\title{
Territoire de recherche
}

Oléagineux, Corps Gras, Lipides. Volume 7, Numéro 6, 461, Novembre - Décembre 2000, Editorial

Auteur(s) : Jean-Claude Icart, .

Résumé : Les questions d'ordre cognitif que posent les choix européens en matière de politique agricole sont plus que jamais à l'ordre du jour. La crise de l'ESB révèle l'importance du travail de recherche à entreprendre sur les problèmes de qualité des produits, de sécurité sanitaire, de bienêtre animal. Avec les notions de développement durable, de valorisation des terroirs, de biodiversité, de coexistence équilibrée entre diverses utilisations du territoire - notions introduites en 1999 par la loi d'orientation agricole -, c'est un champ de réflexion indissociable du précédent qui a été ainsi officiellement ouvert et dont dépendent la définition d'orientations socialement attendues et l'émergence de meilleures réponses aux besoins des marchés.

\section{ARTICLE}

Les questions d'ordre cognitif que posent les choix européens en matière de politique agricole sont plus que jamais à l'ordre du jour. La crise de l'ESB révèle l'importance du travail de recherche à entreprendre sur les problèmes de qualité des produits, de sécurité sanitaire, de bien-être animal. Avec les notions de développement durable, de valorisation des terroirs, de biodiversité, de coexistence équilibrée entre diverses utilisations du territoire - notions introduites en 1999 par la loi d'orientation agricole -, c'est un champ de réflexion indissociable du précédent qui a été ainsi officiellement ouvert et dont dépendent la définition d'orientations socialement attendues et l'émergence de meilleures réponses aux besoins des marchés.

La question des territoires est en effet paradigmatique de toute une époque de la PAC. Autrefois lieux des identités historiques ${ }^{1}$, devenus " objets absents » de la politique européenne, les territoires de l'Union ont été envisagés conformément au schéma porté par le projet de la PAC lui-même, c'està-dire comme simples supports des processus de segmentation et de verticalisation des filières. Soit une dynamique d'" abstraction territoriale $"^{2}$, dont les effets associés ${ }^{3}$ aux performances productives obtenues appellent aujourd'hui la mise en place de nouvelles solidarités entre productions, développement rural et développement durable.

Les acteurs de la gouvernance européenne ne sont évidemment pas seuls concernés par ces problèmes de « représentation » plus ouverts aux dimensions systémiques de l'action...

De nouvelles synthèses sont ici exigées de la recherche agronomique, tant elle s'est également modelée sur cette verticalisation. 
Adapté à ces problématiques complexes l'objectif est désormais pour elle de traiter " les finalités de gestion de l'environnement et de l'espace rural comme des objectifs à part entière et non plus seulement comme des co-produits de recherches axées sur la production ${ }^{4}$.

L'importance des questions méthodologiques est un des éléments qui ressortent de ce dossier constitué autour de ces sujets.

Objet spécifique, non déductible d'une approche in vitro, en fait continuum de dimensions " naturelles " et socio-techniques (de la microbiologie des sols au peuplement, de l'exploitation agricole aux paysages, des bassins versants à l'histoire régionale, etc.), le territoire et sa connaissance impliquent une démarche adaptée. Michel Sebillotte parle ici du territoire comme d'un " construit social " et met au centre de la démarche, y compris pour les questions touchant à l'interdisciplinarité, le développement de relations de partenariat avec les acteurs socioéconomiques concernés et une co-construction collégiale de la ou des dimensions territoriales à traiter. Soit des conclusions qui recoupent celles de la sociologie des sciences et qui conduisent le chercheur à se faire négociateur des problèmes de connaissance, à s'accepter et à s'impliquer dans un processus où se déterminent les acteurs impliqués et leur identité.

Le dépassement de l'« abstraction territoriale ", unissant le contexte et le complexe, trouve ici une voie d'approche.

\section{Références}

${ }^{1}$ Voir Bertrand HERVIEU, Jean VIARD (2001). L'Archipel paysan, la fin de la république agricole, Éditions de l'Aube.

2 Expression de Bertrand HERVIEU. In : Recherches pour et sur le développement territorial, Symposium de Montpellier, 11-12/01/2000. Inra, DADP.

${ }^{3}$ Voir pour un tabeau synthétique de ces effets, Louis Pascal MAHÉ, F ORTALO-MAGNÉ (2001). L'avenir d'un modèle européen de l'espace rural, Paris : Presses de Siences-Po, un ouvrage qui par ailleurs semble abandonner toute idée de « politique agricole » autre qu'environnementale (! ?).

${ }^{4}$ Voir INRA, Document d'orientations 2001-2004. 